This item is the archived peer-reviewed author-version of:

Role of coating-metallic support interaction in the properties of electrosynthesized Rh-based structured catalysts

\title{
Reference:
}

Benito Patricia, de Nolf Wout, Nuyts Gert, Janssens Koen, et al..- Role of coating-metallic support interaction in the properties of electrosynthesized Rh-based structured catalysts

ACS catalysis - ISSN 2155-5435 - 4:10(2014), p. 3779-3790

Full text (Publishers DOI): http://dx.doi.org/doi:10.1021/cs501079k

To cite this reference: http://hdl.handle.net/10067/1222150151162165141 


\section{Role of Coating-Metallic Support Interaction in the Properties of Electrosynthesized}

\section{Rh-Based Structured Catalysts}

Patricia Benito $^{\dagger *}$, Wout de Nolf ${ }^{*^{* *}}$, Gert Nuyts ${ }^{\ddagger}$, Marco Monti ${ }^{\dagger}$, Giuseppe Fornasari ${ }^{\dagger}$,

Francesco Basile ${ }^{\dagger}$, Koen Janssens ${ }^{\ddagger}$, Francesca Ospitali ${ }^{\dagger}$, Erika Scavetta ${ }^{\dagger}$, Domenica Tonelli ${ }^{\dagger}$, Angelo Vaccari ${ }^{\dagger}$

† Dipartimento di Chimica Industriale “Toso Montanari”, ALMA MATER STUDIORUM Università di Bologna, Viale Risorgimento 4, 40136, Bologna, Italy

$\ddagger$ Department of Chemistry, University of Antwerp, Groenenborgerlaan 171, 2020 Antwerp, Belgium

corresponding authors: * patricia.benito3@unibo.it; $* * *$ wout.denolf@ uantwerpen.be 

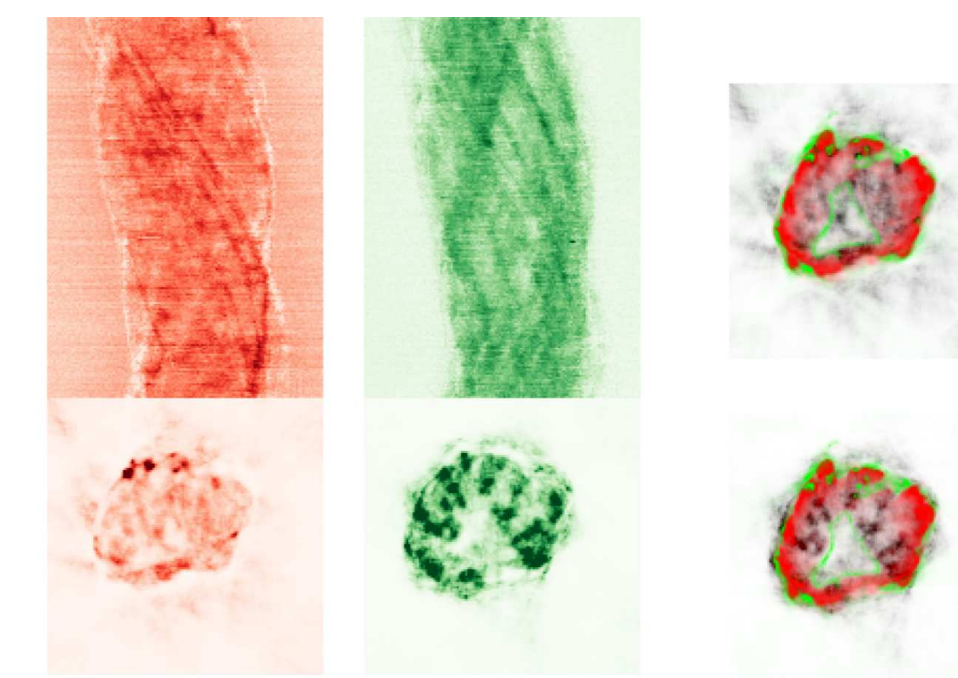

$\mathrm{Fe} / \mathrm{Cr}$

$\mathrm{Fe}+\mathrm{Cr}\left(\mathrm{K}_{\beta}\right)$

$(\alpha+\mathrm{l})-\mathrm{Al}_{2} \mathrm{O}_{3}$

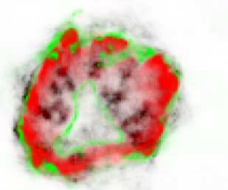

$\mathrm{Cr}_{15.58} \mathrm{Fe}_{7.42} \mathrm{C}_{6}$ $\mathrm{Fe}+\mathrm{Cr}\left(\mathrm{K}_{\beta}\right)$ $(\alpha+\mathrm{l})-\mathrm{Al}_{2} \mathrm{O}_{3}$

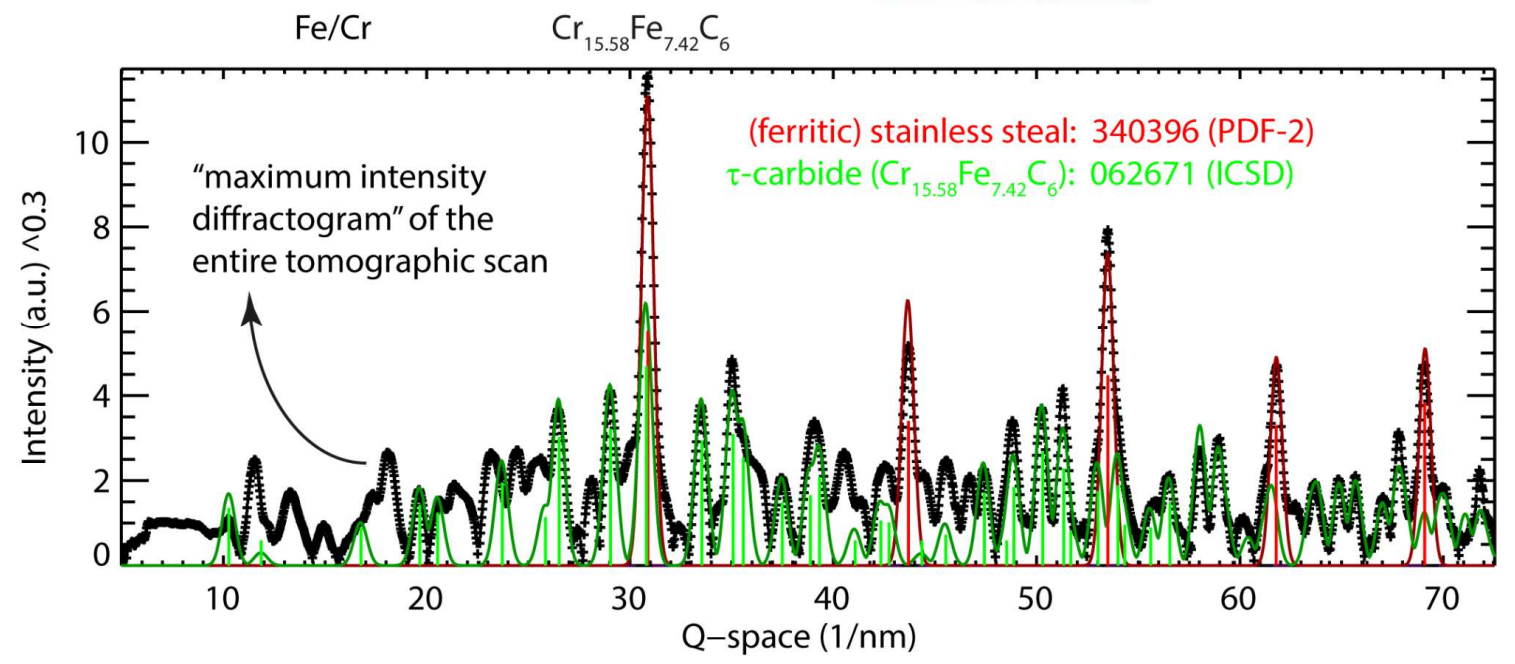

Figure S1. Reconstructions of the Fe/Cr alloy bulk material (stainless steel with ferrite crystal structure) and $\tau$-carbide $\left(\mathrm{Cr}_{23+\mathrm{x}} \mathrm{Fe}_{\mathrm{x}} \mathrm{C}_{6}\right.$ with $\left.\mathrm{x}=7.42\right)$ in a $\mathrm{Rh}_{11.0} \mathrm{Mg}_{70.0} \mathrm{Al}_{19.0}$ strut (state $\mathrm{C}$ ). Translation: $170 \mu \mathrm{m}\left(1.3 \mu \mathrm{m}\right.$ steps); Rotation: $180^{\circ}$ (1 ${ }^{\circ}$ steps); Time: 1 s per point. Comparison with the $\mathrm{Fe}+\mathrm{Cr}$ elemental distribution and the alumina scale (both defining the inner and outer surface of the foam) shows that the reconstruction of $\mathrm{Fe} / \mathrm{Cr}$ and $\tau$-carbide is unreliable. The sinograms are obtained by fitting all diffractograms with a model that includes the $\tau$-carbide (ICSD 062671) and ferrite (AMCSD 0011214) crystal structures, as illustrated for the "maximum intensity diffractogram" of the entire tomographic scan. 


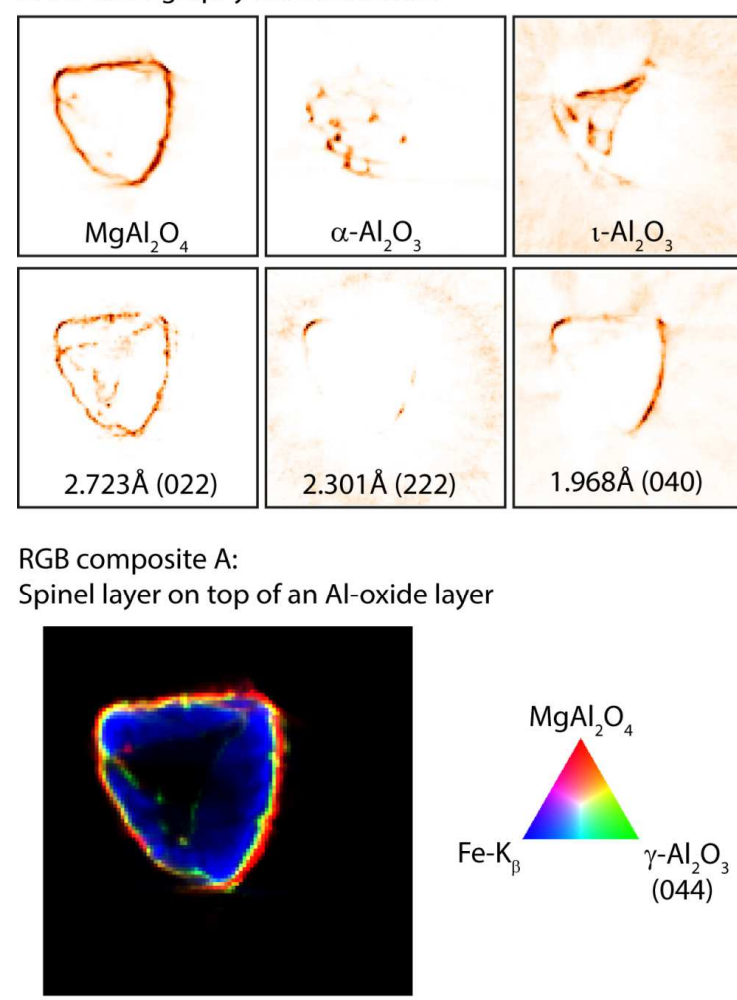

RGB composite B:

Other Al/Cr-oxides formed mostly at the inner cavity surface
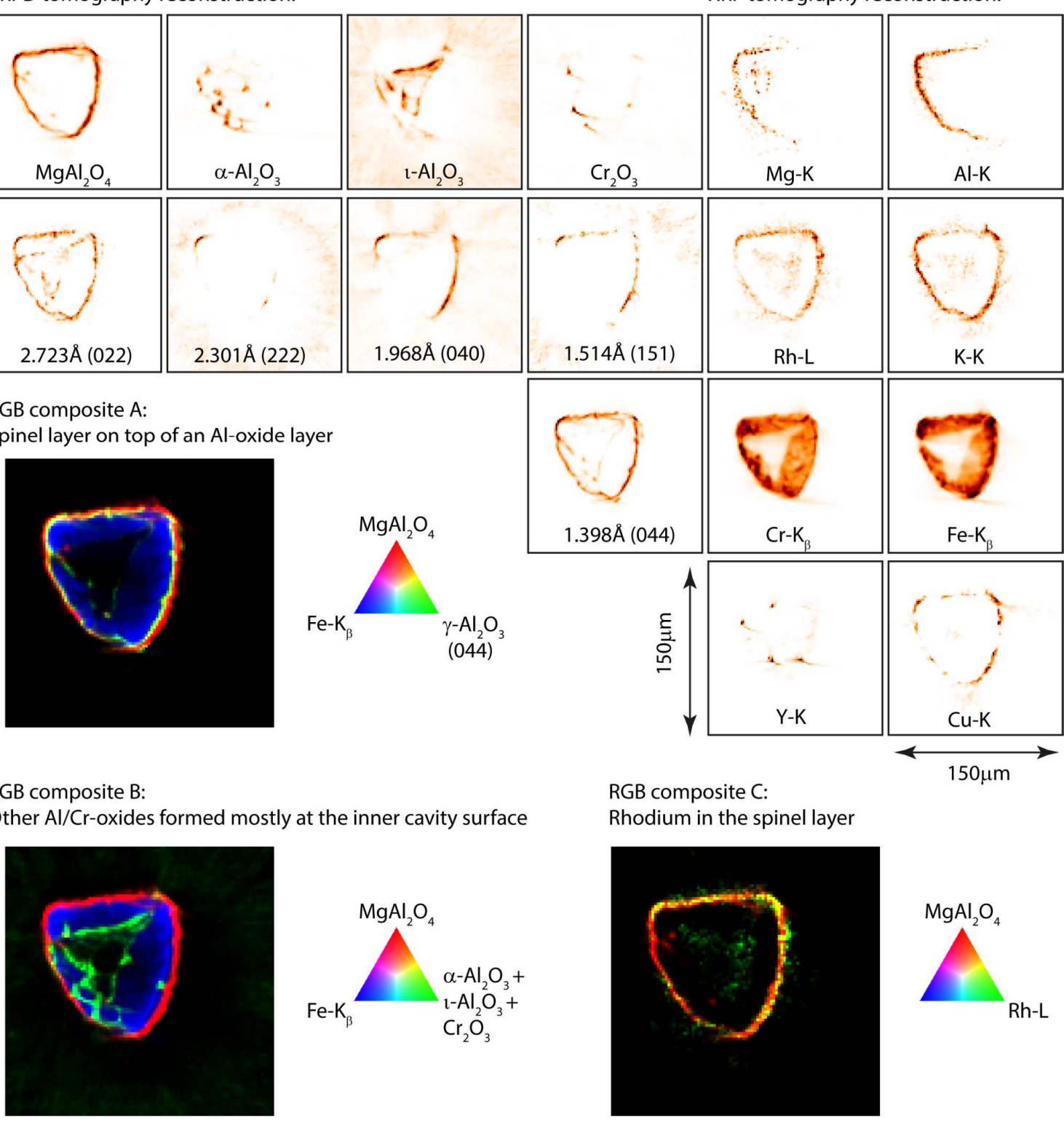

RGB composite C:

Rhodium in the spinel layer
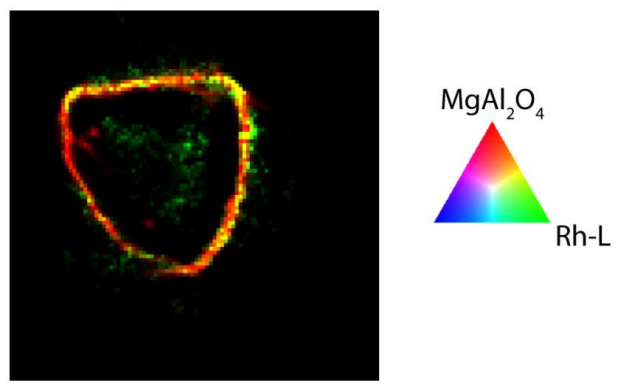

Figure S2. Elemental and crystalline distributions in a virtual cross-section of a calcined $\mathrm{Rh}_{5.0} \mathrm{Mg}_{70.0} \mathrm{Al}_{25.0}$ strut (state C). Translation: $150 \mu \mathrm{m}$ (1.5 $\mu \mathrm{m}$ steps): Rotation: $180^{\circ}\left(1.2^{\circ}\right.$ steps); Time: $1 \mathrm{~s}$ per point. The unidentified reflections may be attributed to $\gamma-\mathrm{Al}_{2} \mathrm{O}_{3}$. $\mathrm{RGB}$ composite maps show that roughly two layers are formed on top of the foam surface: a spinel layer (containing rhodium) and an oxide layer which takes the form of $\gamma-\mathrm{Al}_{2} \mathrm{O}_{3}$ at the outer foam surface and $\alpha / \mathrm{l}-\mathrm{Al}_{2} \mathrm{O}_{3} / \mathrm{Cr}_{2} \mathrm{O}_{3}$ at the inner cavity surface. 


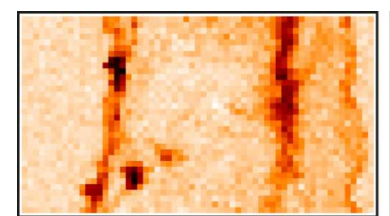

$\mathrm{MgAl}_{2} \mathrm{O}_{4}$

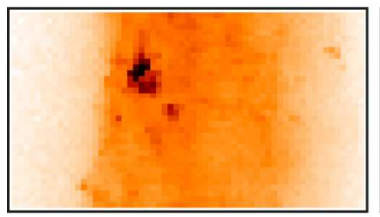

$\mathrm{Fe} / \mathrm{Cr}$
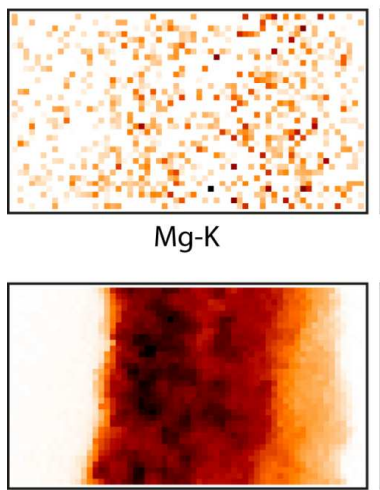

$\mathrm{Cr}-\mathrm{K}_{\beta}$

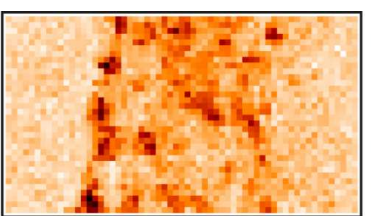

$\alpha-\mathrm{Al}_{2} \mathrm{O}_{3}$

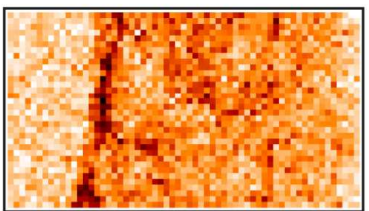

$3.633 \AA$

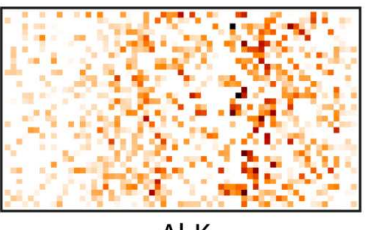

Al-K

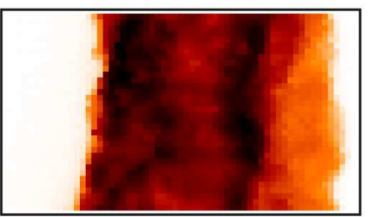

$\mathrm{Fe}-\mathrm{K}_{\beta}$

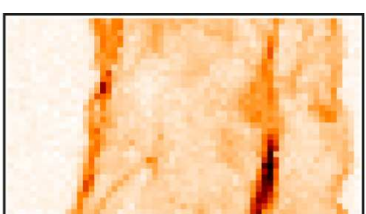

$1-\mathrm{Al}_{2} \mathrm{O}_{3}$

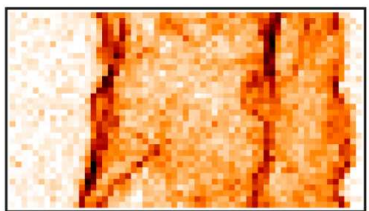

$1.397 \AA ̊(044)$

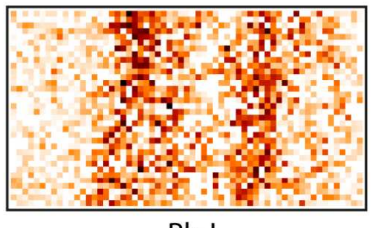

Rh-L

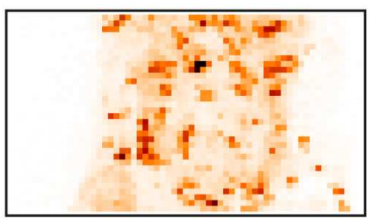

Y-K
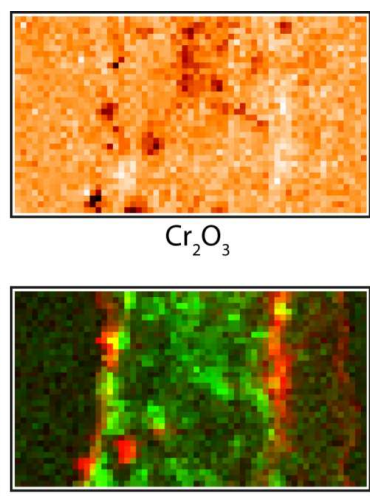

$\mathrm{MgAl}_{2} \mathrm{O}_{4} \quad \alpha-\mathrm{Al}_{2} \mathrm{O}_{3}$

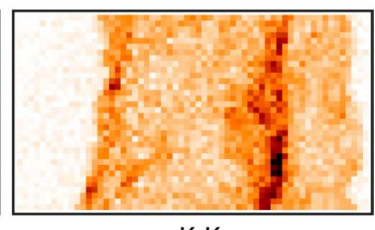

K-K

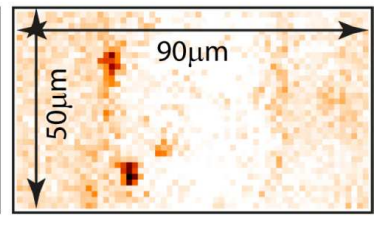

$\mathrm{Cu}-\mathrm{K}$

Figure S3. Projected elemental and crystalline distributions $(1.5 \times 1.5 \mu \mathrm{m}$ step size for a $1.5 \times$ $3.0 \mu \mathrm{m}$ beam size) in a calcined $\mathrm{Mg}_{70.0} \mathrm{Al}_{30.0}$ strut (state C). Horizontal: $90 \mu \mathrm{m}$ (1.5 $\mu \mathrm{m}$ steps); Vertical: $50 \mu \mathrm{m}(1.5 \mu \mathrm{m}$ steps); Time: $1 \mathrm{~s}$ per point. The unidentified $1.397 \AA$ Bragg peak might be attributed to the most intense reflection (044) of $\gamma-\mathrm{Al}_{2} \mathrm{O}_{3}$. 


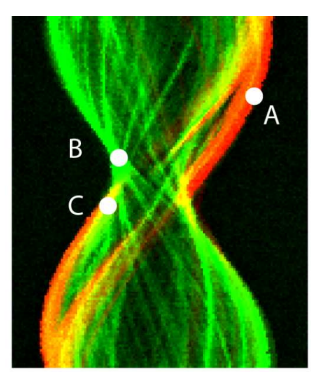

Mg-Al spinel with Al-Rh substitution and without inversion: $[\mathrm{Mg}]^{8 \mathrm{a}}\left[\mathrm{Rh}_{\mathrm{x}} \mathrm{Al}_{1-\mathrm{x}}\right]_{2}{ }^{16 \mathrm{~d}} \mathrm{O}_{4}$
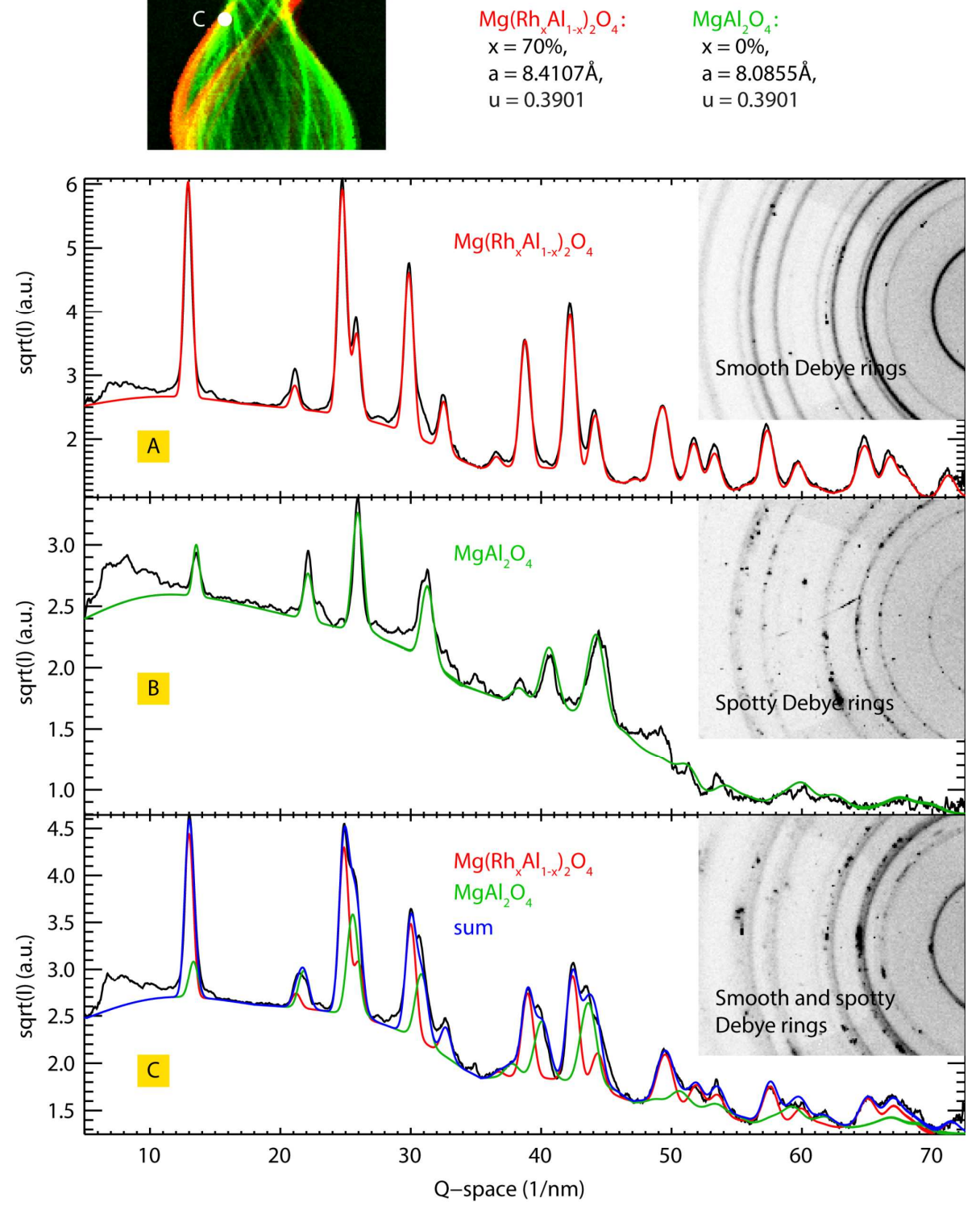

Figure S4. Diffractograms of $\mathrm{Mg}\left(\mathrm{Rh}_{\mathrm{x}} \mathrm{Al}_{1-\mathrm{x}}\right)_{2} \mathrm{O}_{4}(\mathrm{~A}), \mathrm{MgAl}_{2} \mathrm{O}_{4}(\mathrm{~B})$ and of a mixture of the two (C) selected from a tomographic scan of $\mathrm{Rh}_{13.6} \mathrm{Mg}_{86.4}$ (state C). Translation: $190 \mu \mathrm{m}(1.5 \mu \mathrm{m}$ steps); Rotation: $180^{\circ}\left(1.2^{\circ}\right.$ steps); Time: $1 \mathrm{~s}$ per point. Not only the lattice parameter (and hence Bragg peak shifts) and atomic substitution (and hence Bragg peak intensities) allow for a distinction, but also the appearance of the Debye rings (smooth or spotty) and the peak intensity ratios. 

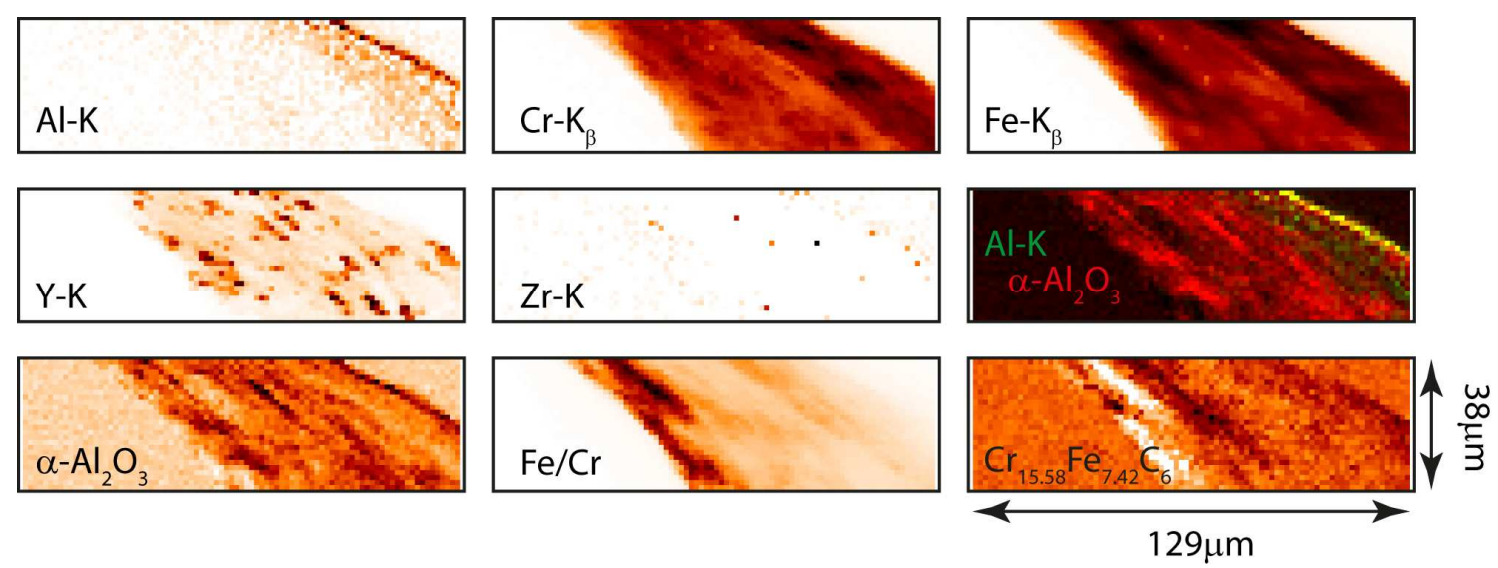

Figure S5. Projected elemental and crystalline distributions in calcined bare foam strut.

Horizontal: $129 \mu \mathrm{m}(1.5 \mu \mathrm{m}$ steps); Vertical: $38 \mu \mathrm{m}(1.5 \mu \mathrm{m}$ steps); Time: $1 \mathrm{~s}$ per point. 


\section{$\mu$ XRF/XANES.}

\section{Rhodium speciation by XANES.}

The applied energy step size was adapted to slope at different energies in the XANES spectrum, meaning that the smallest step size is chosen around the Rh- $\mathrm{L}_{3}$ edge $(2920 \mathrm{eV}$ $2954 \mathrm{eV}: 2 \mathrm{eV}, 2955 \mathrm{eV}-2980 \mathrm{eV}: 1 \mathrm{eV}, 2980.5 \mathrm{eV}-3000 \mathrm{eV}: 0.5 \mathrm{eV}, 3000.25 \mathrm{eV}-3014$ eV: $0.25 \mathrm{eV}, 3014.5 \mathrm{eV}-3075 \mathrm{eV}: 0.5 \mathrm{eV}$ and $3076 \mathrm{eV}-3135 \mathrm{eV}: 1 \mathrm{eV})$. A $1.6 \mathrm{~s}$ acquisition time was employed for each energy, resulting in $\sim 14$ min recording time for one spectrum. Several repeats with a maximum of 3 were recorded for each measured point in order to reduce noise.

$\mathrm{Rh}-\mathrm{L}_{3}$ spectra typically show a white-line in the range of 3005-3007 eV (depending on the oxidation state) due to the electron transition from $2 p_{3 / 2}$ to $4 d_{3 / 2}$ and $4 d_{5 / 2}{ }^{1}$ whose intensity is known to be correlated with the number of $4 d$-holes. ${ }^{2}$ A decrease of the white-line intensity is thus observed in the more reduced samples. ${ }^{3}$ Peaks at energies above the white-line are attributed to transitions to $\mathrm{pd}, \mathrm{df}, \mathrm{f}$ and $\mathrm{df}$ orbitals. ${ }^{1}$ Since linear combination fitting (LCF) of unknown sample spectra with reference spectra is used to determine the Rh speciation, nine reference compounds were measured. Three compounds in which $\mathrm{Rh}$ is present in a spineltype phase: calcined $\mathrm{RhMgAlO}_{4}, \mathrm{Rh}_{11} \mathrm{Mg}_{70} \mathrm{Al}_{19}$ and $\mathrm{Rh}_{5} \mathrm{Mg}_{70} \mathrm{Al}_{25}$, two hydroxide compounds (starting products): $\mathrm{Rh}_{11} \mathrm{Al}_{89}$ and $\mathrm{Rh}_{11} \mathrm{Mg}_{70} \mathrm{Al}_{19}$ (hydrotalcite), $\mathrm{Rh}$ metal, $\mathrm{Rh}_{2} \mathrm{O}_{3}$ (as received and calcined at $900^{\circ} \mathrm{C}$ ) and calcined $\mathrm{MgRh}_{2} \mathrm{O}_{4}$ were measured. All measured reference spectra were corrected for self-absorption using the built-in function of ATHENA, influencing only the intensity of the white line. Some reference spectra, however, did not show significant differences relative to others: all three $\mathrm{Rh}$ spinel compounds, both hydroxide compounds and both $\mathrm{Rh}_{2} \mathrm{O}_{3}$ compounds essentially show an identical XANES spectrum, reducing the number of reference spectra to five. PCA analysis of all sample spectra discussed in this article revealed that 3 components are needed to sufficiently reconstruct all unknown XANES 
spectra. Interestingly, none of the combinations of the described five spectra resulted in satisfactory LCF for all measured samples. However, when including a spectrum of the coated foam after CPO tests into the fitting model (state E), together with calcined $\mathrm{Rh}_{5.0} \mathrm{Mg}_{70.0} \mathrm{Al}_{25.0}$ (state $\mathrm{C}$, presence determined by XRPD analysis) and $\mathrm{Rh}_{2} \mathrm{O}_{3}$ (reference compound, presence determined by TPR), it was possible to adequately describe all other sample spectra. 
Table S1. Summary of the Rh speciation of all measured samples determined by means of linear combination fitting of unknown spectra with three reference spectra: $\mathrm{Rh}_{5.0} \mathrm{Mg}_{70.0} \mathrm{Al}_{25.0}$ spinel, $\mathrm{Rh}_{2} \mathrm{O}_{3}$ and a spectrum of a $\mathrm{Rh}_{11.0} \mathrm{Mg}_{70.0} \mathrm{Al}_{19.0}$ strut (state $\mathrm{E}$ ). The percentages represent the amount of $\mathrm{Rh}$ present as each given reference compound. Since spectrum $5 c_{-} 1$ is used as a reference to fit all other spectra no results can be given for this spectrum.

\begin{tabular}{|c|c|c|c|c|}
\hline Sample & & $\mathrm{SA} \mathrm{dRh}_{5.0} \mathrm{Mg}_{70.0} \mathrm{Al}_{25.0}$ & Sample5c_1 & $\mathrm{SA} \mathrm{dRh}{ }_{2} \mathrm{O}_{3}$ \\
\hline $\mathrm{Rh}_{11.0} \mathrm{Mg}_{70.0} \mathrm{Al}_{19.0}$ State $\mathrm{C} \_1$ & $5 a \_1$ & 0.41 & 0.60 & 0.00 \\
\hline $\mathrm{Rh}_{11.0} \mathrm{Mg}_{70.0} \mathrm{Al}_{19.0 \_}$State $\mathrm{C} \_2$ & $5 \mathrm{a} \_2$ & 0.30 & 0.68 & 0.02 \\
\hline $\mathrm{Rh}_{11.0} \mathrm{Mg}_{70.0} \mathrm{Al}_{19.0 \_}$State $\mathrm{C} \_3$ & $5 \mathrm{a} \_3$ & 0.62 & 0.28 & 0.10 \\
\hline $\mathrm{Rh}_{11.0} \mathrm{Mg}_{70.0} \mathrm{Al}_{19.0 \_}$State $\mathrm{C} \_4$ & $5 \mathrm{a} \_4$ & 0.25 & 0.75 & 0.00 \\
\hline $\mathrm{Rh}_{11.0} \mathrm{Mg}_{70.0} \mathrm{Al}_{19.0 \_}$State C_5 & $5 \mathrm{a} \_5$ & 0.49 & 0.51 & 0.00 \\
\hline $\mathrm{Rh}_{11.0} \mathrm{Mg}_{70.0} \mathrm{Al}_{19.0 \_}$State C_6 & $5 \mathrm{a} \_6$ & 0.09 & 0.91 & 0.00 \\
\hline $\mathrm{Rh}_{11.0} \mathrm{Mg}_{70.0} \mathrm{Al}_{19.0 \_}$State $\mathrm{C}_{-} 7$ & $5 a_{-} 7$ & 0.37 & 0.43 & 0.19 \\
\hline $\mathrm{Rh}_{11.0} \mathrm{Mg}_{70.0} \mathrm{Al}_{19.0 \_}$State $\mathrm{C} \_8$ & $5 \mathrm{a} \_8$ & 0.36 & 0.32 & 0.32 \\
\hline $\mathrm{Rh}_{11.0} \mathrm{Mg}_{70.0} \mathrm{Al}_{19.0 \_}$State C_9 & $5 \mathrm{a}_{-} 9$ & 0.23 & 0.64 & 0.13 \\
\hline $\mathrm{Rh}_{11.0} \mathrm{Mg}_{70.0} \mathrm{Al}_{19.0 \_}$State $\mathrm{C} \_10$ & $5 a_{-} 10$ & 0.27 & 0.44 & 0.30 \\
\hline $\mathrm{Rh}_{11.0} \mathrm{Mg}_{70.0} \mathrm{Al}_{19.0 \_}$State $\mathrm{C}$ & $5 a_{-} 11$ & 0.32 & 0.50 & 0.18 \\
\hline $\mathrm{Rh}_{11.0} \mathrm{Mg}_{70.0} \mathrm{Al}_{19.0 \_}$State $\mathrm{D} \_1$ & & 0.19 & 0.81 & 0.00 \\
\hline $\mathrm{Rh}_{11.0} \mathrm{Mg}_{70.0} \mathrm{Al}_{19.0 \_}$State $\mathrm{D} \_2$ & & 0.06 & 0.94 & 0.00 \\
\hline $\mathrm{Rh}_{11.0} \mathrm{Mg}_{70.0} \mathrm{Al}_{19.0 \_}$State $\mathrm{D} \_3$ & & 0.13 & 0.86 & 0.00 \\
\hline $\mathrm{Rh}_{11.0} \mathrm{Mg}_{70.0} \mathrm{Al}_{19.0 \_}$State $\mathrm{D} \_4$ & $5 \mathrm{~b} \_1$ & 0.11 & 0.89 & 0.00 \\
\hline $\mathrm{Rh}_{11.0} \mathrm{Mg}_{70.0} \mathrm{Al}_{19.0 \_}$State D_5 & $5 b \_2$ & 0.17 & 0.83 & 0.00 \\
\hline $\mathrm{Rh}_{11.0} \mathrm{Mg}_{70.0} \mathrm{Al}_{19.0 \_}$State D_6 & & 0.39 & 0.61 & 0.00 \\
\hline $\mathrm{Rh}_{11.0} \mathrm{Mg}_{70.0} \mathrm{Al}_{19.0 \_}$State $\mathrm{D}_{-}$7 & & 0.32 & 0.65 & 0.04 \\
\hline $\mathrm{Rh}_{11.0} \mathrm{Mg}_{70.0} \mathrm{Al}_{19.0 \_}$State $\mathrm{D} \_8$ & & 0.22 & 0.78 & 0.00 \\
\hline $\mathrm{Rh}_{11.0} \mathrm{Mg}_{70.0} \mathrm{Al}_{19.0 \_}$State D_9 & & 0.15 & 0.85 & 0.00 \\
\hline $\mathrm{Rh}_{11.0} \mathrm{Mg}_{70.0} \mathrm{Al}_{19.0 \_}$State $\mathrm{D}_{-} 10$ & & 0.20 & 0.80 & 0.00 \\
\hline $\mathrm{Rh}_{11.0} \mathrm{Mg}_{70.0} \mathrm{Al}_{19.0 \_}$State E_1 & & 0.27 & 0.70 & 0.03 \\
\hline $\mathrm{Rh}_{11.0} \mathrm{Mg}_{70.0} \mathrm{Al}_{19.0 \_}$State E_2 & & 0.06 & 0.94 & 0.00 \\
\hline $\mathrm{Rh}_{11.0} \mathrm{Mg}_{70.0} \mathrm{Al}_{19.0 \_}$State E_3 & & 0.14 & 0.85 & 0.01 \\
\hline $\mathrm{Rh}_{11.0} \mathrm{Mg}_{70.0} \mathrm{Al}_{19.0 \_}$State E_4 & & 0.00 & 1.00 & 0.00 \\
\hline $\mathrm{Rh}_{11.0} \mathrm{Mg}_{70.0} \mathrm{Al}_{19.0 \_}$State E_5 & $5 c_{-} 1$ & n.a. & n.a. & n.a. \\
\hline $\mathrm{Rh}_{11.0} \mathrm{Mg}_{70.0} \mathrm{Al}_{19.0}$ State E_6 & $5 c \_2$ & 0.00 & 1.00 & 0.00 \\
\hline $\mathrm{Rh}_{11.0} \mathrm{Mg}_{70.0} \mathrm{Al}_{19.0 \_}$State E_7 & $5 c \_3$ & 0.00 & 1.00 & 0.00 \\
\hline $\mathrm{Rh}_{11.0} \mathrm{Mg}_{70.0} \mathrm{Al}_{19.0 \_}$State $\mathrm{E} \_8$ & $5 c \_4$ & 0.00 & 1.00 & 0.00 \\
\hline $\mathrm{Rh}_{13.6} \mathrm{Mg}_{86.4 \_}$State $\mathrm{C} \_1$ & & 0.39 & 0.61 & 0.00 \\
\hline $\mathrm{Rh}_{13.6} \mathrm{Mg}_{86.4 \_ \text {State }} \mathrm{C} \_2$ & & 0.28 & 0.56 & 0.16 \\
\hline $\mathrm{Rh}_{13.6} \mathrm{Mg}_{86.4 \_}$State $\mathrm{C} \_3$ & & 0.76 & 0.24 & 0.00 \\
\hline $\mathrm{Rh}_{13.6} \mathrm{Mg}_{86.4 \_}$State $\mathrm{C} \_4$ & & 0.64 & 0.36 & 0.00 \\
\hline $\mathrm{Rh}_{13.6} \mathrm{Mg}_{86.4 \_}$State C_5 & & 0.88 & 0.12 & 0.00 \\
\hline
\end{tabular}




\section{REFERENCES}

(1) Sham, T. K. Phys. Rev. B 1985, 31, 1888-1902.

(2) de Groot, F. M. F.; Hu, Z. W.; Lopez, M. F.; Kaindl, G.; Guillot, F.; Trone, M. J. Chem. Phys. 1994, 101, 6570-6576.

(3) Shimizu, K.-i.; Oda, T.; Sakamoto, Y.; Kamiya, Y.; Yoshida, H.; Satsuma, A. Appl. Catal. B: Env. 2012, 111-112, 509-514. 